\title{
Online Low-Price Guarantees-A Real Options Analysis
}

\author{
Benjamin Marcus \\ Sawyer School of Management, Suffolk University, Boston, Massachusetts 02116, bmarcus@ suffolk.edu \\ Chris K. Anderson \\ School of Hotel Administration, Cornell University, Ithaca, New York 14853, cka9@cornell.edu
}

\begin{abstract}
A common practice among large retailers is the low-price guarantee, rebating consumers if they find an identical product cheaper elsewhere. This provides consumers with some level of comfort in their purchase decision. A similar low-price guarantee is provided by numerous service industries that allow reservation of capacity, yet do not penalize the consumer for failure to keep that reservation-examples include hotels and car rental. Given that a consumer is not required to keep the reservation, they may make another reservation, either at a competing firm or the same firm, if future prices decline. The increasing availability of pricing information on the Internet affords consumers the opportunity to be more strategic in their purchasing behavior. As consumers, we are able to quickly and easily check prices from numerous service or goods providers. The ease of price information potentially makes these guarantees very costly to the service or good provider. We analyze the implied costs associated with these guarantees by making analogies to financial options. Motivation for this research comes from a large car rental firm, Dollar Thrifty Automotive Group Inc., that considered offering a low-price guarantee to all consumers that book a reservation though their website.
\end{abstract}

Subject classifications: finance: asset pricing; inventory/production: perishable items; transportation: automobile. Area of review: New/Nontraditional Areas.

History: Received July 2004; revisions received March 2005, August 2005; accepted August 2005.

\section{Introduction}

In November 1997, Daimler Chrysler Motors Corporation divested itself of Dollar Thrifty Automotive Group (DTAG). Currently, DTAG owns two major car rental brands: Dollar Rent-A-Car Systems, Inc. and Thrifty RentA-Car System, Inc. Based in Tulsa, Oklahoma, DTAG has grown over the past five years to approximately 460 worldwide locations in 26 different countries, including 260 in the United States. The fleet size now exceeds 200,000 vehicles, of which nearly 100,000 are in the United States. Their primary focus is on the price-sensitive leisure traveler in the United States. DTAG's revenues have grown steadily over the past five years and now exceed US $\$ 1.3$ billion per year.

DTAG can also claim that they have become one of the most, if not the most, profitable car rental companies in the world. "For the third consecutive year, DTAG boasts the highest profit margin of all car rental companies primarily serving airport-arriving customers" (www.DTAG.com). The majority of DTAG's revenues are from corporately owned outlets for which they actively manage prices, with a smaller percentage of sales coming from independently operated franchise outlets. In 1998 they began an implementation of a Talus Rental Car Revenue Management System. They have spent significant time and effort both in utilizing this system and in developing several other complementary revenue management tools and practices. The revenue management department balances a conservative approach while strategically capitalizing on science-based systematic approaches to pricing.

DTAG concentrates on the value-conscious traveler in the North American market, typically catering to the leisure traveler or other consumers who are more price sensitive. This target market dictates that DTAG must be a lowcost operator to remain competitive because the margins on these rentals are thin. Due to the low margins DTAG maintains, operational efficiency is one of their key success factors.

DTAG relies heavily on revenue management systems to change up to 100,000 rental rates daily, propelling it to one of the most profitable companies in the industry. The power to change many rates helps maintain a competitive advantage in an industry where rates are changing constantly as companies jockey for the consumer's business.

\subsection{Revenue Management}

Airlines have successfully used dynamic pricing and revenue management to increase their profitability. Fares on a flight can differ dramatically. Segmentation of customers is achieved, for instance, by restrictions on refunds, requiring a Saturday night stay or advance purchase. Even a small percentage increase in revenue per flight can have important effects on an airline's profitability. In fact, American Airlines attributes over $\$ 500$ million in additional annual 
revenues to its revenue management activities (Smith et al. 1992). The impact from revenue management is reinforced by a recent McKinsey \& Co. study analyzing Compustat data for 1,000 companies, indicating that price has the most dramatic impact on profits (versus costs or volumes), as a $1 \%$ increase in price (revenues) generates a $7.4 \%$ increase in profits (Anthes 1998). It is not unusual for an airline to open a flight for bookings up to a year in advance. The selective availability of fare classes and the management of that availability are what constitute revenue management.

The concept of revenue management is not specific to the airline industry. It has been applied to numerous other businesses that have similar characteristics, including car rental, broadcasting, cruise lines, Internet service provision, lodging, and railways. There are several common characteristics of yield management practice (Kimes 1989): ability to segment markets, relatively fixed capacity, perishable inventory, product sold in advance, fluctuating demand, and low marginal sales cost/high marginal capacity change cost.

Since the early 1990s the car rental industry has also given increasing attention to revenue management. Most work in the area of car rental revenue management has been the application of airline revenue management methods to the car rental setting. Carroll and Grimes (1995) and Geraghty and Johnson (1997) provide accounts of the state of the art in car rental revenue management.

One of the distinct differences of car rental revenue management from its application to airlines is the degree to which prices change. Airlines typically have several discrete price classes, across which prices are fairly constant. Car rental firms also have discrete price classes for the different types of cars (e.g., economy versus luxury) and different durations of rental (e.g., daily versus weekly), but actively change the prices within these classes on a daily basis. Rental companies continually change prices to maximize profits. On a particular day, if DTAG has a large number of cars available they may discount the rental rate to take advantage of the excess supply, and increase or hold steady on rates if demand is exceeding expectations.

The car rental market is fiercely competitive, with both Budget and ANC Rental (parent of Alamo and National) filing for bankruptcy protection in 2002. The industry saw sharp increases in consumer demand in the late 1990s into 2000 , but has seen a dramatic decline in demand since September 11th, 2001, with relatively flat growth expected into 2005 (Datamonitor 2003). The competitive nature of this industry in combination with flat growth prospects sees the industry becoming more cost conscious.

\subsection{Online Sales}

The recent proliferation of the Internet has been fully grasped by the travel industry. Airlines have been among the first to exploit the opportunities presented by the digital economy. Each year an ever-increasing fraction of their tickets are sold over the Internet. Revenues from Internet bookings totaled $\$ 276$ million in 1996 , tripled to $\$ 827$ in
Table 1. Typical DTAG distribution channel volume (2003) and associated costs.

\begin{tabular}{lcc}
\hline Channel & $\begin{array}{c}\text { \% of total } \\
\text { bookings }\end{array}$ & $\begin{array}{c}\text { Cost to DTAG } \\
\text { per rental }\end{array}$ \\
\hline Walkups & 14 & 5 \\
800 number & 16 & $\$ 6.00$ \\
Dollar.com, Thrifty.com & 27 & $\$ 0.75$ \\
Travel agent bookings & 13 & 15 \\
Third-party websites* & 30 & $15 \%$ \\
\hline
\end{tabular}

*Expedia, Orbitz, Travelocity, etc.

1997, and topped \$3.3 billion in 1999 (Miller 1999). Recent surveys have online airline ticket sales at $\$ 16$ billion in 2001 - forecast to reach $\$ 40$ billion by 2007 . While initially the advent of online sales was seen as a cost-effective way to reach customers, the recent success of third-party websites such as Orbitz, Travelocity, and Expedia has in fact increased sales and distribution costs for many firms. For instance, DTAG pays commissions of approximately $15 \%$ on bookings received via these third-party websites. Table 1 summarizes DTAG's selling costs and fraction of sales by channel.

Online sales through DTAG's own websites, Dollar.com and Thrifty.com, are very cost effective, whereas a $15 \%$ commission on rentals that average $\$ 40$ per day is considerable.

\subsection{Cancellations, No-Shows, and Corporate Rates}

The airline industry's first big gains from revenue management came through the use of overbooking, with overbooking, representing about $40 \%$ of revenue management benefits (cross 1995). Overbooking is the process of overselling seats to compensate for those travelers who fail to show at departure time. Augmenting overbooking, airlines offer numerous types of tickets that are nonrefundable if the consumer fails to make the flight. The car rental industry actively overbooks their fleets, primarily because the industry typically does not penalize consumers for failure to keep reservations. Historically, the car rental industry has had cancellation rates in the neighborhood of $20 \%-30 \%$. The lack of penalties for failure to keep a reservation means a consumer may continue to shop for cheaper rates even after they have made a reservation. Consumers may have multiple reservations for the same location and arrival day, resulting in numerous data difficulties for the car rental firm, not to mention hidden costs.

A similar hidden cost to the car rental firm is associated with corporate and governmental rates. Firms or groups often get access to discounted rates as a result of memberships or contracts, yet members of these groups are not obligated to accept these rates-they may shop for lower rates. 


\subsection{The Low-Price Guarantee}

In an effort to encourage bookings through their own websites (www.Dollar.com and www.Thrifty.com), DTAG was considering offering a low-price guarantee. The guarantee was twofold, where first, DTAG's own sites would have the lowest posted rate of any website for a DTAG rental vehicle; and second, if rates declined from the time you reserved your vehicle until the time you picked it up (and you booked on Dollar or Thrifty.com), DTAG would refund you the difference. The second aspect of the guarantee is not new to marketers, and is referred to as most-favored-customer protection (Png 1991, Cooper 1986). Similarly, the offer is not that different from current promotions offered by numerous hoteliers. Marriott International recently announced a best-rate pledge, where they would rebate consumers $25 \%$ if they found a lower rateeither through a Marriott channel or third-party source-for a Marriott room within 48 hours after making a reservation through Marriott (Binkley 2003).

While the offer seems generous, the low-price guarantee is already embedded in their cancellation policy (or lack thereof) and corporate rate agreements, as consumers have the option to continue to shop for lower rates-the guarantee simply makes this option explicit. Before proceeding with the promotion, DTAG needed to value this option.

\section{Evaluating the Promotion}

The following section outlines the pricing of the low-price guarantee. The low-price guarantee may be valued either empirically using historic data or analytically using a model of rental prices. Similar to stock price option valuation, the following details an analytical model of the option. Later we compare analytical and empirical valuations of the guarantee.

\subsection{Real-Option Analysis}

In recent years, a new theory of pricing and operating assets in the face of uncertainty, and in the presence of some flexibility in operating strategies, has been developed. This is the theory of real options (Dixit and Pindyck 1994, Amram and Kulatilaka 1999). The theory of real options in turn uses the framework of modern financial options pricing to frame and solve its problems. For general mathematical references on options pricing see Merton (1990), Hull (2003), and Wilmott (2000).

Different types of real options exist, depending on the flexibility possessed by the business operator. For example, consider a project with known start-up costs that may be initiated any time in the next year. Such a project may be modelled as an American call option-the real-option holder is allowed to "buy into" the project or exercise his option on any date in the next year. Pricing such an option requires a decision about the optimal way in which to exercise it. The two problems are solved in tandem.
Table 2. DTAG percentage of reservations by days prior to pickup during summer 2003.

\begin{tabular}{cccc}
\hline Days out & $\begin{array}{c}\text { Percentage } \\
\text { of total }\end{array}$ & Days out & $\begin{array}{c}\text { Percentage } \\
\text { of total }\end{array}$ \\
\hline 0 & 14 & 16 & 1 \\
1 & 22 & 17 & 1 \\
2 & 11 & 18 & 1 \\
3 & 6 & 19 & 1 \\
4 & 5 & 20 & 1 \\
5 & 4 & 21 & 1 \\
6 & 4 & 22 & 1 \\
7 & 3 & 23 & 1 \\
8 & 3 & 24 & 1 \\
9 & 2 & 25 & 1 \\
10 & 2 & 26 & 1 \\
11 & 2 & 27 & 0.5 \\
12 & 2 & 28 & 0.5 \\
13 & 2 & 29 & 0.5 \\
14 & 2 & 30 or more & 1.5 \\
15 & 2 & & \\
\hline
\end{tabular}

Note. 0-walkups.

\subsection{The Low-Price Guarantee}

The booking of a reservation provides the consumer with the right, with no obligation, to rent the vehicle-they may continue to shop for lower rates. The low-price guarantee formalizes this option, as the consumer will pay the minimum of the rate they lock in at time of reservation and all posted rates between that time and when they are due to pick up their vehicle. The payout to the consumer, who reserves the car at rate $P_{0}$, will be

option $=\max \left[0, P_{0}-m_{T_{0}}^{T}\right]$

where $m_{T_{0}}^{T}$ is the minimum of posted rental prices from the time of reservation $T_{0}$ until vehicle pickup at time $T$.

Car rental firms start taking reservations for vehicles as early as a year in advance of pickup, with the bulk of the reservations being made within a few weeks of pickup. Table 2 provides a representative breakdown of bookings by days prior to pickup.

Figure 1 displays actual daily rates for a single product (one car class, fixed rental duration, single location) for Monday pickups during June 2003. The figure contains the rates for Mondays in June as individual series, as well as the daily average (solid line) of the four Mondays. The figure provides some insight into the value of the option to the consumer because while on average rates tend to increase as the pickup day approaches, there are several instances when rates decline.

\subsection{Pricing Model}

The application of financial option-pricing approaches to the valuation of real assets requires that the value of the underlying variable follow a random process that is exogenous to the holder. The value of a financial option is linked 
Figure 1. Daily rental rates for Monday pickups during July 2003.

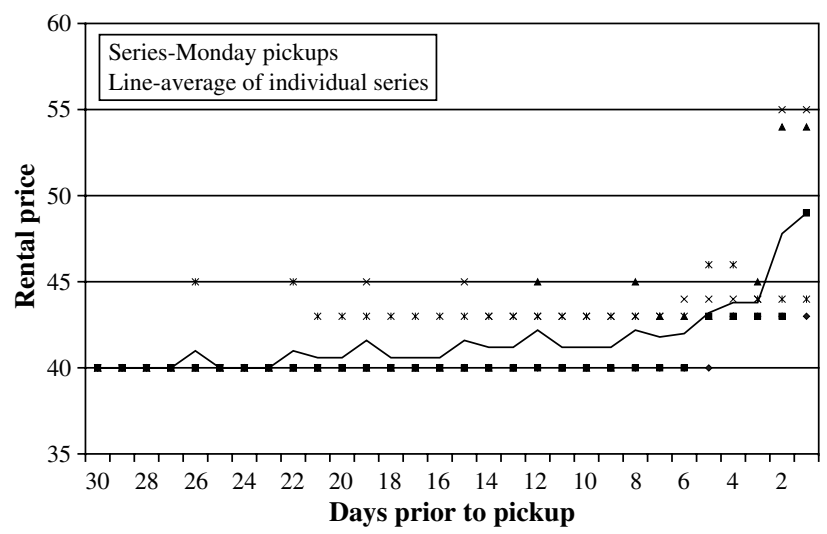

to the price of a stock, the value or price of which is dictated by the market, not the owner of the option. This requirement often limits the accurate application of realoptions analysis.

DTAG primarily rents vehicles at airport locations, and as a result they cannot create demand through pricing. Rather, they capture a portion of the business at these locations, where demand is predominantly the result of air travel. Price changes do generate subtle changes in demand, but what is more important is one car rental firm's price against its competition's (Blair 2002). Figure 2 is a simplified illustration of price elasticity within the car rental industry. Price elasticity, the percentage change in quantity per percentage change in price, describes the sensitivity of sales volume to price changes. In Figure 2, demand is relatively inelastic over price changes from $P_{2}$ to $P_{3}$, as very little additional sales result $\left(\Delta_{Q 2}\right.$ is small), while very elastic from $P_{1}$ to $P_{2}\left(\Delta_{Q 1}\right.$ is quite large). Over the range $P_{1}$ to $P_{2}$, the price has crossed a competitor' price $P_{C}$, generating the volume increase (decrease), whereas from $P_{2}$ to $P_{3}$ it is still priced between two competitors, or already has the lowest price.

Figure 2. Car rental price elasticity.

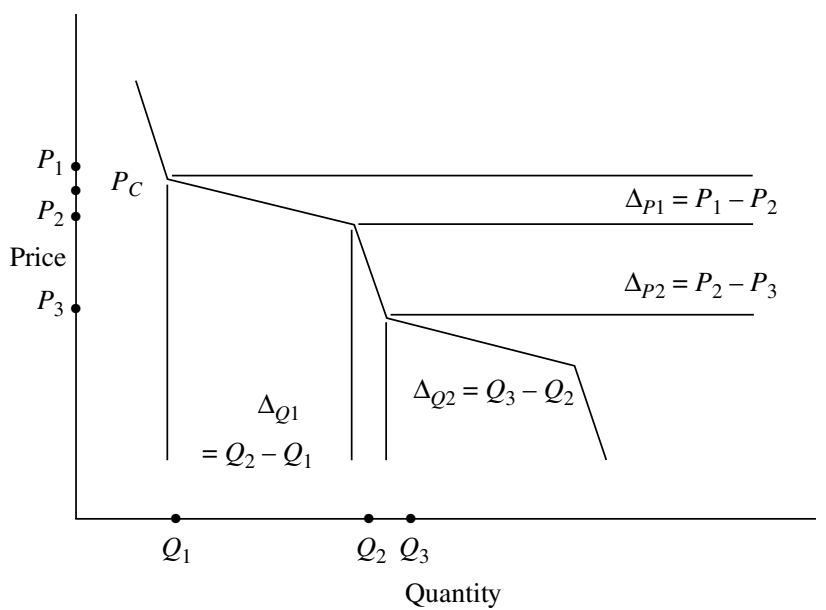

The net result of this price behavior is that DTAG has little pricing power and is largely a price-taker, having to price relative to the competition. As a price-taker, the value of the low-price guarantee (LPG) is dictated by market forces, somewhat outside the control of DTAG. Blair and Anderson (2002) give an accurate account of pricing activity at DTAG, with Anderson et al. (2004) outlining the impact of pricing on car rental revenue management.

Given the price response shown in Figure 2, we will model price as an exogenous random process. In essence, we are modeling the rental car firm as a pure price-taker, unable to arbitrarily price, but having to price relative to the competition. The valuation of the option requires an understanding of the evolution of rental prices from the day of reservation through to pickup. We look at several approaches to modeling rental car prices, using geometric Brownian motion (GBM) as the foundational underlying process.

Figure 3 lays out the timing of the option valuation. A reservation is made at price $P_{0}$ at time $T_{0}$ for pickup of a vehicle at time $T$ in the future; we look to value the potential rebate to consumers at some time $t$ between $T_{0}$ and $T$. We specify $m_{T_{0}}^{t}$ as the lowest posted rental price observed from time $T_{0}$ to $t$.

Equation (1) indicates that the option is a function of the reservation price $P_{0}$ and the minimum price between reservation and pickup $m_{T_{0}}^{T}$. For car rental firms of the size of DTAG, renting nearly 100,000 vehicles daily, the option can be restated as

option $=e^{-\rho T} E\left[\max \left(0, P_{0}-\left[m_{T_{0}}^{T} \mid P_{0}\right]\right)\right]$,

where $E\left[m_{T_{0}}^{T} \mid P_{0}\right]$ is the expected minimum price from reservation until pickup. The option is then the expected discounted future cash flows (discounting at some riskmeasure-specific discount rate $\rho$ ) associated with the guarantee, noting that if the guarantee is paid it occurs at pickup.

Equation (2) holds for any price process, and only requires the calculation of the expectation of the minimum.

2.3.1. Rental Prices Following Geometric Brownian Motion. GBM is the mainstay in valuing financial options when stock prices are the underlying asset. GBM seems

Figure 3. Option timing and rental prices.

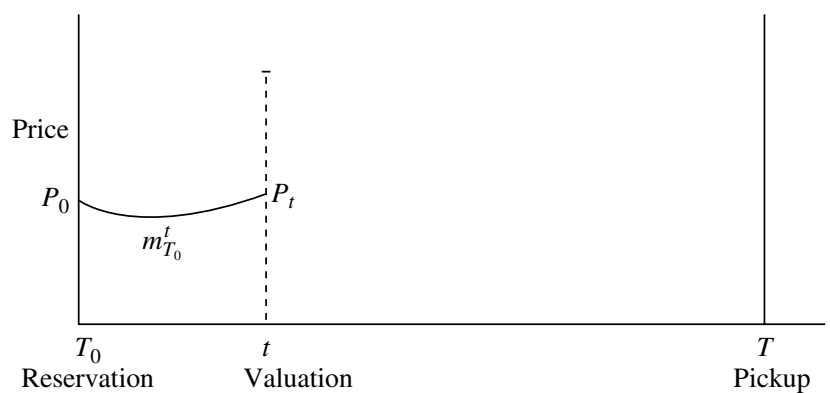


a logical model for rental prices, because, similar to stock prices, rental prices tend to increase in time, yet exhibit short-term fluctuations. The basic assumption in this framework is that prices follow a lognormal random process, with a known mean (drift) and standard deviation (volatility). We will value the LPG option with an underlying following GBM for both constant and time-dependent parameters, under both real-world (risk-adjusted) and riskneutral measures. For all four cases, $P_{0}$ is the strike price, $m_{T_{0}}^{t}$ is the minimum price between time zero (booking), $T_{0}$, and the current time, $t$. For ease of notation, we define $\tau$ as time to expiry, where $\tau=T-t$.

GBM Model 1A. Constant drift and volatility under a real-world (risk-adjusted) measure.

Assume that rental price $P$ follows the lognormal process

$d P=\mu P d t+\sigma P d Z$

where $\mu$ and $\sigma$ are the constant real-world drift and volatility, respectively, and $d Z$ is an increment of a standard Wiener process. As in Equation (2), we write the option value as an expectation,

$p^{P}\left(P_{t}, m_{T_{0}}^{t}, \tau\right)=e^{-\rho \tau} E^{P}\left[\max \left(0, P_{0}-\left[m_{T_{0}}^{T} \mid P_{0}\right]\right)\right]$,

where $\rho$ is a firm-specific risk-adjusted discount rate, and the expectation is taken under the probability-generating measure $P$, the real-world drift and volatility. The option value has a closed-form expression (see the appendix for derivation): for $m_{T_{0}}^{t} \geqslant P_{0}$, we have that

$$
\begin{aligned}
& p^{P}\left(P_{t}, m_{T_{0}}^{t}, \tau\right) \\
& =-P_{t} e^{\left(\left(\sigma^{2} / 2\right)+\mu-\rho\right) \tau} N(-d)+e^{-\rho \tau} P_{0} N(-d+\sigma \sqrt{\tau}) \\
& +\frac{\sigma^{2} e^{-\rho \tau}}{\sigma^{2}+2 \mu} P_{t}\left\{\left(\frac{P_{0}}{P_{t}}\right)^{\left(\sigma^{2}+2 \mu\right) / \sigma^{2}} N\left(-d+\frac{\left(\sigma^{2}+2 \mu\right) \sqrt{\tau}}{\sigma}\right)\right. \\
& \left.-e^{\left(\left(\sigma^{2} / 2\right)+\mu\right) \tau} N(-d)\right\},
\end{aligned}
$$

where

$d=\frac{\ln \left(P_{t} / P_{0}\right)+\left(\sigma^{2}+\mu\right) \tau}{\sigma \sqrt{\tau}}$,

and for $m_{T_{0}}^{t}<P_{0}$, we have that

$$
\begin{aligned}
& p^{P}\left(P_{t}, m_{T_{0}}^{t}, \tau\right) \\
& =e^{-\rho \tau}\left(P_{0}-m_{T_{0}}^{t}\right)+m_{T_{0}}^{t} e^{-\rho \tau} N\left(-d^{\star}+\sigma \sqrt{\tau}\right) \\
& -P_{t} e^{\left(\left(\sigma^{2} / 2\right)+\mu-\rho\right) \tau} N\left(-d^{\star}\right)+\frac{\sigma^{2} e^{-\rho \tau}}{\sigma^{2}+2 \mu} \\
& \cdot P_{t}\left\{\left(\frac{m_{T_{0}}^{t}}{P_{t}}\right)^{\left(\sigma^{2}+2 \mu\right) / \sigma^{2}} N\left(-d^{\star}+\frac{\left(\sigma^{2}+2 \mu\right) \sqrt{\tau}}{\sigma}\right)\right. \\
& \left.-e^{\left(\left(\sigma^{2} / 2\right)+\mu\right) \tau} N\left(-d^{\star}\right)\right\}
\end{aligned}
$$

where

$d^{\star}=\frac{\ln \left(P_{t} / m_{T_{0}}^{t}\right)+\left(\sigma^{2}+\mu\right) \tau}{\sigma \sqrt{\tau}}$.

Equations (5) and (6) are of a form similar to financial fixed-strike lookback options (Conze and Viswanathan 1991, Goldman et al. 1979), with differences reflecting the absence of the typical hedging arguments used in financial option pricing.

GBM Model 1B. Constant drift and volatility under a risk-neutral measure.

It is possible to modify Equation (3) to accommodate risk-neutral pricing (Trigeorgis 1996). To do so, we must adjust the real-world drift $\mu$ downward by subtracting an appropriate risk premium, and then price the option as though the world were risk neutral. Let $\lambda=(\mu-r) / \sigma$, where $r$ is the risk-free interest rate. The parameter $\lambda$, known as the market price of risk, represents the rate of additional return required by the market for bearing an additional unit of risk. Then, the risk premium associated with the rental price is $\lambda \sigma$, and we obtain the risk-neutral price process,

$d P=r P d t+\sigma P d Z$

by setting $\mu=r$. For this process, we again write the option value as an expectation,

$p^{Q}\left(P_{0}, m_{T_{0}}^{T}, T\right)=e^{-r T} E^{Q}\left[\max \left(0, P_{0}-\left[m_{T_{0}}^{T} \mid P_{0}\right]\right)\right]$,

where $E^{Q}$ is the expectation under the risk-neutral probability-generating measure $Q$. This leads to an optionpricing formula similar to Equations (5) and (6), and can be obtained by substituting $r$ for $\mu$ and $\rho$.

If drift and volatility are not constant, then the above models may either undervalue or overvalue the option. We believe that at times rental prices may exhibit some timedependent behavior. We now extend the models in Equations (3) and (7) to capture the dynamics of time-dependent parameters.

GBM Model 2A. Time-dependent drift and volatility under a real-world (risk-adjusted) measure.

Let $\mu(t)$ and $\sigma(t)$ be the real-world drift and volatility at time $t$, respectively. The lognormal price process for these time-dependent parameters becomes

$d P=\mu(t) P d t+\sigma(t) P d Z$.

This process does not admit a closed-form solution for the option value. Instead, we employ Monte Carlo simulation (see, for example, Trigeorgis 1996) to calculate the expected value of the LPG. Monte Carlo simulation is advantageous in this setting because it can neatly capture the path-dependent nature of this option and provide an 
estimate of the option value over the entire booking horizon for each sample path.

To begin, we divide the booking horizon into $N$ equal subintervals, so that each period $i=0,1, \ldots, N$ is of length $\Delta t=T / N$. We generate a sample price path from the discrete stochastic process

$P_{i}=\mu_{i-1} P_{i-1} \Delta t+\sigma_{i-1} P_{i-1} \sqrt{\Delta t} \tilde{\varepsilon}_{i}$,

where $P_{0}=P\left(T_{0}\right), \mu_{i}=\mu(i \Delta t), \sigma_{i}=\sigma(i \Delta t)$, and each $\tilde{\varepsilon}_{i}$ is a random sample from a standardized normal distribution. For each sample path, define $m_{i}$ as $\min P_{k}$ over $k \epsilon[i, N]$. Then, the option value $v_{i}$ (at time $T$ ) for each period becomes

$v_{i}=\max \left(P_{i}-m_{i}, 0\right)$.

The Monte Carlo option value is obtained by averaging over $M$ such path realizations, and discounting back to time $T_{0}$. Let $v_{i}^{j}$ be the realized option value in period $i$ for path $j, j=1, \ldots, M$. Then, the Monte Carlo value of the LPG for each period, priced under the real-world measure, is

$V_{i}=e^{-\rho\left(T-T_{i}\right)} \frac{1}{M} \sum_{j=1}^{M} v_{i}^{j}$

GBM Model 2B. Time-dependent drift and volatility under a risk-neutral measure.

The price process of Equation (9) can be adjusted to allow risk-neutral pricing, as done in the constant parameter case:

$d P=r P d t+\sigma(t) P d Z$.

Again, we can use Monte Carlo simulation to obtain estimates of the LPG value over the booking horizon. Replacing $\mu(t)$ and $\rho$ with $r$ in Equations (10)-(12), enables Monte Carlo pricing of the option $V$ under the risk-neutral measure.

\subsection{Model Fitting and Parameter Estimation}

GBM seems to be a reasonable model for rental car prices because they tend to increase as the pickup date approaches, and are always positive. GBM for rental prices implies that returns on prices (increases period over period) are normally distributed. Continuously compounded returns in rental prices are calculated as $\ln \left(P_{t} /\left(P_{t}-1\right)\right)$. Tests for normality in returns were performed visually in conjunction with chi square tests. Figure 4 displays a sample histogram and Q-Q plot for overnight rates on intermediatesize cars at a specific location for the last week prior to pickup. Chi-square tests were performed for two separate locations for both short and long-term rental periods for four types of rental vehicles with chi-square values ranging from 0.8 to 4.8 ( $p$-values 0.98 to 0.44 ); here we do not wish
Figure 4. Daily returns on overnight rentals for intermediate-size cars, last week prior to pickup.

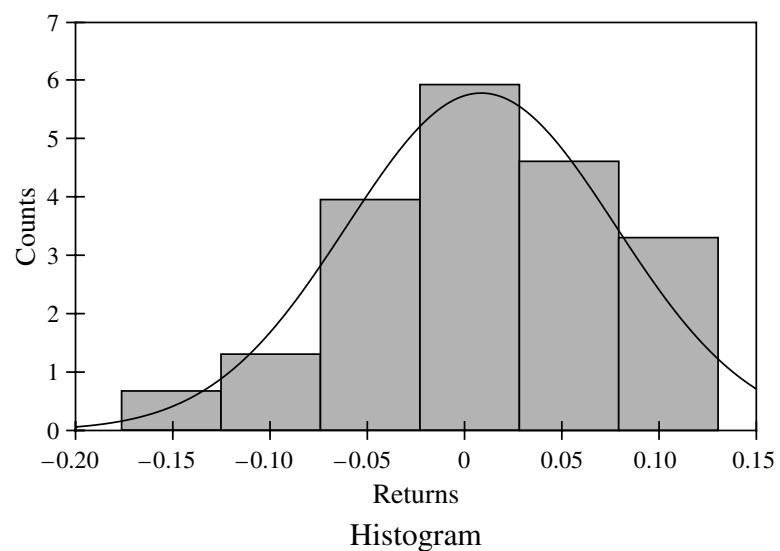

Histogram

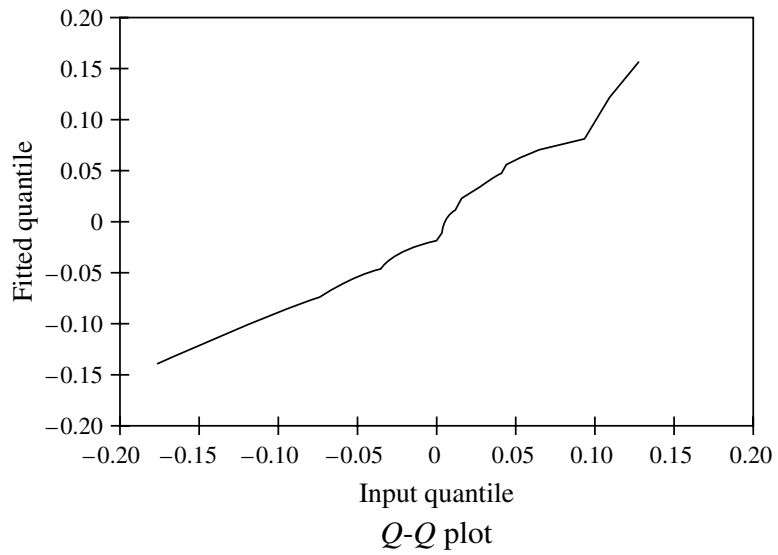

to reject the null. Generally speaking, while some combinations of rental duration, location, and car type are less adequately represented by normal distributions, under most circumstances GBM seems reasonable. Fortunately, combinations that fit the assumptions best, like the one depicted in Figure 4, are also those where valuation is most critical because overnight rentals account for the majority of rentals and exhibit the most volatility in prices. The data from these two rental locations is used throughout the paper for parameter estimation and illustration. Time-dependent estimates of $\mu$ and $\sigma$ are calculated from samples using daily prices. For example, estimation of drift and volatility in the last period prior to vehicle pickup, $\mu(1)$ and $\sigma(1)$, can be obtained from samples of $\ln \left(P_{0} / P_{1}\right)$, over a series of pickup dates, where $P_{0}$ are prices at pickup and $P_{1}$ are prices for vehicles reserved one day in advance provides. The average of the sample provides an estimate of $\mu(1)-\sigma^{2}(1) / 2$ and the standard deviation of the sample an estimate of $\sigma(1)$.

\subsection{Assessing the Guarantee}

As an illustration of the potential cost of the option, Figure 5 shows a set of hypothetical guarantee payoffs (i.e., calculating payoffs with actual rental rates). The figure illustrates the value (cost) of the low-price guarantee to DTAG for overnight rentals (economy and full-size 
Figure 5. Low-price guarantee option value during summer 2003.

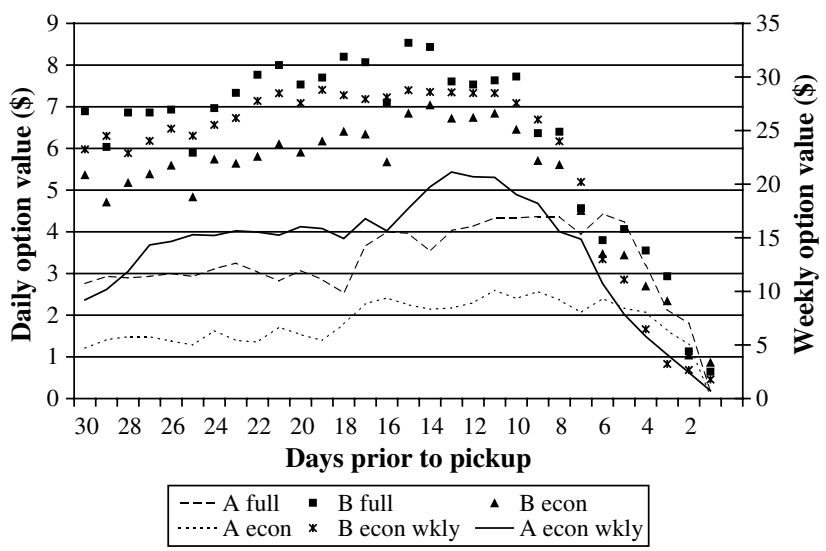

vehicles), as well as weekly rentals (economy) if it had been in place in the summer of 2003 for two cities (A and $\mathrm{B})$.

The empirical evaluation of the guarantee payoffs as illustrated in Figure 5 is data intensive and only indicative of potential historical payout. The methods developed in $\$ 2.3 .1$ can be used to evaluate the option under a wide variety of pricing environments.

Figure 6 compares the different valuation approaches to the empirical value for economy overnight rentals at location A. The GBM approaches, both analytical and numerical, are performed under real-world measures (GBM Models $1 \mathrm{~A}$ and $2 \mathrm{~A}$ in $\$ 2.3 .1$ ) as growth and volatility may be estimated from data, with DTAG providing a riskadjusted discount rate. Given that price changes at DTAG are made daily with data for parameter estimation available at the daily level, $\Delta t$ for Monte Carlo valuation was one day. Growth and volatility were estimated from daily returns in rental prices. The options are valued for the last 30 days prior to vehicle pickup, with a different set of growth and volatility values used for each day in the Monte Carlo formulation.

Figure 6. Comparison of low-price guarantee option values for economy daily rentals.

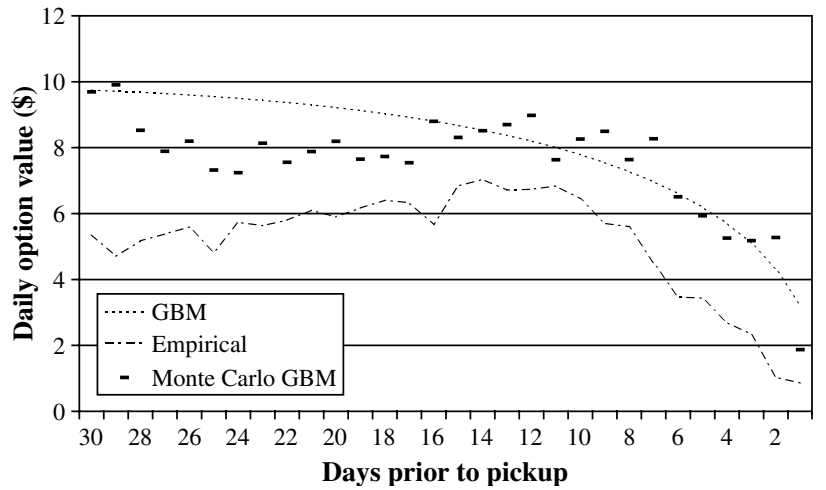

Monte Carlo valuation was performed with 5,000 replications. Given that the Monte Carlo approach will undervalue the option (a continuously sampled minimum is never bigger than the discretely sampled minimum), a control variate form of variance reduction is used to correct for sampling frequency, similar to the simulation of Asian options (Wilmott 2000). Using constant growth and volatility parameters, the true value $\left(V^{\prime}\right)$ of the option is known from Equation (5). The discretely sampled values of the option $\left(V_{s}\right)$ can then be compared to the true value, the difference a result of sampling frequency. The option value with time-dependent parameters $\left(C_{s}\right)$ can then be adjusted based on the sampling error,

$C_{s}=C_{s}\left(1+\frac{V^{\prime}-V_{s}}{V^{\prime}}\right)$

(using a set of common random numbers for both $V_{s}$ and $C_{s}$ ). Figure 6 illustrates that the GBM approach may overvalue the option early on in the process, with the time-dependent parameter Monte Carlo approach exhibiting similar behavior to the empirical values, but generally higher valuation.

The cost of low-price guarantee to DTAG can be determined by calculating the weighted average value of the option. The weighted average value is calculated by taking the fraction of consumers who purchase each day prior to pickup (Table 2) and multiplying that by the value of the option (as a function of time) and summing these products. Table 3 summarizes the weighted average cost of the guarantee under GBM for numerous parameter sets (constant parameters) for options in place 30 days prior to vehicle pickup.

Table 3 uses six sets of parameter values. The first four parameter pairs represent modest values for the average and standard deviations of growth in car rental prices over the 30-day window just prior to vehicle pickup. The last two parameter pairs are extreme values calculated from 2003 rental car data. The values represent the lowest and highest observed growth rates, 0 and 0.4 , respectively; and the lowest and highest standard deviations, 0.2 and 0.8 , respectively. The first set $(0,0.8)$ represents a no-growth setting with high volatility and produces very high costs for the guarantee. The second set $(0.4,0.2)$ represents high steady growth, producing lower option value.

Table 3. Low-price guarantee costs (\$) under GBM.

\begin{tabular}{lc}
\hline $\begin{array}{l}\text { Growth } \\
(\mu, \sigma)\end{array}$ & Option value \\
\hline$(0.1,0.1)$ & 1.02 \\
$(0.1,0.3)$ & 3.61 \\
$(0.3,0.1)$ & 0.68 \\
$(0.3,0.3)$ & 4.19 \\
$(0,0.8)$ & 8.66 \\
$(0.4,0.2)$ & 1.84 \\
\hline
\end{tabular}


Figure 7. Break-even option value as fraction at DTAG websites and fraction that switch.

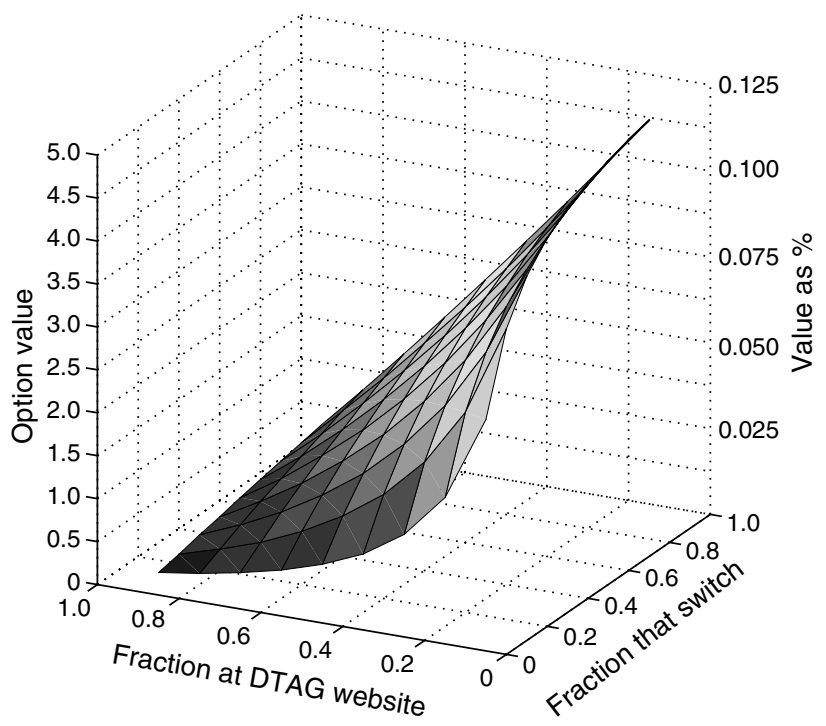

The purpose for proposing the low-rate guarantee was to entice online consumers to make reservations on the lowercost DTAG websites. Figure 7 illustrates sample break-even option values as a function of the percent of Internet customers that are using DTAG's site (i.e., $1-x$ are using a third-party site) and the fraction of third-party consumers that switch once the guarantee is offered. The figure is based on a rental with an average rate of $\$ 40$. The trade-off is the $\$ 5.25$ saved in booking fees $(\$ 0.75$ versus commission of \$6.00) versus the options paid out, i.e.,

savings $=$ costs,

$$
\begin{aligned}
& (15 \% * \$ 40.00-\$ 0.75) * F_{3 r d} * F_{\text {switch }} \\
& \quad=\text { option } *\left[\left(1-F_{3 r d}\right)+F_{3 r d} * F_{\text {switch }}\right], \\
& \text { option }=\frac{\$ 5.25 * F_{3 r d} * F_{\text {switch }}}{\left[\left(1-F_{3 r d}\right)+F_{3 r d} * F_{\text {switch }}\right]},
\end{aligned}
$$

where $F_{3 r d}$ is the fraction of Internet reservations made on third-party sites (Expedia, Orbitz, etc.) and $F_{\text {switch }}$ is the fraction of that group that switches to either Dollar or Thrifty.com.

The option values as summarized in Table 3 indicate a wide range of valuations with the prices under GBM. As illustrated in Figure 6, GBM potentially overvalues the option early on, with the differences between approaches less dramatic nearing vehicle pickup. Given that the bulk of reservations occur within the last week of booking, the guarantee value under GBM provides a basis for assessing the low-price guarantee. Under extreme parameter pairs, GBM indicates costs ranging from $\$ 1.84$ to $\$ 8.66$, with reasonable values of $\$ 3-\$ 4$.

This option would be provided to all customers that booked on DTAG's websites. Figure 7 illustrates that to break even almost all of the traffic from the third-party websites would have to move to DTAG's sites to compensate. Under the current consumer booking structure, the cost of the option seems prohibitive given that $50 \%$ of the online bookings are already on a DTAG site, and these consumers would receive the option even though they were already booking on the DTAG site prior to undertaking the incentive program. Given DTAG's current breakdown of roughly half of the Internet traffic at either Dollar.com or Thrifty.com with the other half at third-party websites, option values must be less than $\$ 2.00$ to break even, even if all Internet traffic moves to a DTAG site. The guarantee potentially becomes a useful incentive in the case that DTAG continues to lose bookings to third-party websites, with the break-even value approaching the $\$ 5.25$ in savings. In this case, the option is justified to induce switching to the DTAG site.

\section{Conclusions}

The insight provided by valuing the option was twofold. First, the costs associated with rebating consumers does not significantly outweigh the fees associated with third-party booking. This assessment was based solely on assuming a constant market share for DTAG, although it is possible that such a program may elicit switching behavior from other car rental firms to DTAG. It is anticipated, given the competitive nature of car rental, that any successful program of stealing business would quickly be replicated.

Second, bringing the idea forward and pricing it has educated company executives on the consumer value associated with costless cancellations and corporate rates. Knowledge of the associated costs potentially enables the creation of new (discounted) products that have cancellation fees or require nonrefundable prepayment.

The development of analytical approaches to valuing either the option or costless cancellations now provides a mechanism to include these "costs" in current revenue management and pricing practices. It is now possible to include the costs associated with price decreases in future pricing decisions, whether these costs are direct or indirect.

The guarantee valuation under GBM is also of general interest as more firms look to offer price protection, whether they are service firms (Marriott), general retail (big-box stores), or manufacturers offering meet or release contracts.

Presently DTAG is deeply discounting rates, by as much as $20 \%$, at Thrifty and Dollar.com (compared with posted rates on other channels) in an effort to sway reservation traffic to their own websites. The secondary $z$-axis in Figure 7 represents the break-even discounts (assuming average rates of \$40) as a function of channel makeup and switching behavior. Clearly, discounts in excess of $5 \%$ or $6 \%$ are losing money. While the guarantee appears to be expensive, it clearly is less detrimental than deep discounts. The low-price guarantee potentially offers consumers perceived value and comfort in their reservation decision and 
can be marketed; as a result, it continues to receive discussion from senior management. The pricing of the guarantee both empirically and analytically has removed considerable uncertainty about the potential risks associated with offering the rate pledge.

\section{Appendix. Risk-Adjusted Option Price Derivation}

In this appendix, we provide the derivation for the optionpricing formula in Equations (5) and (6). This is the option price under a firm-specific risk measure, where $\mu$ and $\sigma$ are the real-world drift and volatility of $\ln P$, respectively. Recall the notation from $\$ 2.3 .1: P_{0}$ is the strike price, $m_{T_{0}}^{t}$ is the minimum price between time zero (booking), $T_{0}$, and the current time, $t$. We define $\tau$ as time to expiry: $\tau=$ $T-t$.

$$
\begin{aligned}
p^{P}\left(P_{t}, m_{T_{0}}^{t}, \tau\right) \\
=e^{-\rho \tau} E^{P}\left[\max \left(P_{0}-\min \left(m_{T_{0}}^{t}, m_{t}^{T}\right), 0\right)\right] \\
=e^{-\rho \tau}\left\{E^{P}\left[\max \left(P_{0}-m_{T_{0}}^{t}, 0\right) \mid m_{T_{0}}^{t} \leqslant m_{t}^{T}\right]\right. \\
\left.\quad+E^{P}\left[\max \left(P_{0}-m_{t}^{T}, 0\right) \mid m_{T_{0}}^{t}>m_{t}^{T}\right]\right\} .
\end{aligned}
$$

The first term, indicating that the lowest price has already been reached, becomes

$$
\begin{aligned}
& E^{P}\left[\max \left(P_{0}-m_{T_{0}}^{t}, 0\right) \mid m_{T_{0}} \leqslant m_{t}^{T}\right] \\
& = \begin{cases}\left(P_{0}-m_{T_{0}}^{t}\right) P\left(m_{T_{0}} \leqslant m_{t}^{T}\right), & m_{T_{0}}^{t}<P_{0}, \\
0, & m_{T_{0}}^{t} \geqslant P_{0}\end{cases} \\
& = \begin{cases}\left(P_{0}-m_{t_{0}}^{T}\right)\left[N\left(\frac{\ln \left(P_{t} / m_{t_{0}}^{T}\right)+\mu \tau}{\sigma \sqrt{\tau}}\right)-\left(\frac{m_{T_{0}}^{t}}{P_{t}}\right)^{2 \mu / \sigma^{2}}\right. \\
\left.\cdot N\left(\frac{\ln \left(m_{T_{0}}^{t} / P_{t}\right)+\mu \tau}{\sigma \sqrt{\tau}}\right)\right], & m_{T_{0}}^{t}<P_{0} \\
0, & m_{T_{0}}^{t} \geqslant P_{0},\end{cases}
\end{aligned}
$$

where

$$
N\left(\frac{\ln \left(P_{t} / m_{t_{0}}^{T}\right)+\mu \tau}{\sigma \sqrt{\tau}}\right)-\left(\frac{m_{T_{0}}^{t}}{P_{t}}\right)^{2 \mu / \sigma^{2}} N\left(\frac{\ln \left(m_{T_{0}}^{t} / P_{t}\right)+\mu \tau}{\sigma \sqrt{\tau}}\right)
$$

is the probability that prices will not decrease below $m_{T_{0}}^{t}$ (more specifically, that returns will not be sufficiently low to reach lower prices) as derived in Harrison (1985).

The second term becomes

$$
\begin{aligned}
E^{P}[\max & \left.\left(P_{0}-m_{t}^{T}, 0\right) \mid m_{T_{0}}^{t}>m_{t}^{T}\right] \\
= & \begin{cases}\int_{-\infty}^{\ln \left(m_{T_{0}}^{t} / P_{t}\right)}\left(P_{0}-P_{t} e^{y}\right) g(y) d y, & m_{T_{0}}^{t}<P_{0}, \\
\int_{-\infty}^{\ln \left(P_{0} / P_{t}\right)}\left(P_{0}-P_{t} e^{y}\right) g(y) d y, & m_{T_{0}}^{t} \geqslant P_{0},\end{cases}
\end{aligned}
$$

where, for

$y=\ln \frac{m_{T_{0}}^{t}}{P_{t}} \leqslant 0$,

the distribution function $g(y)$ is

$$
\begin{aligned}
g(y)= & \frac{\partial}{\partial y}\left[1-P\left(y_{T} \geqslant y\right)\right] \\
= & \frac{\partial}{\partial y}\left[N\left(\frac{-y+\mu \tau}{\sigma \sqrt{\tau}}\right)-e^{2 \mu y / \sigma^{2}} N\left(\frac{y+\mu \tau}{\sigma \sqrt{\tau}}\right)\right] \\
= & \frac{1}{\sigma \sqrt{\tau}} n\left(\frac{-y+\mu \tau}{\sigma \sqrt{\tau}}\right)+\frac{2 \mu}{\sigma^{2}} e^{2 \mu y / \sigma^{2}} N\left(\frac{y+\mu \tau}{\sigma \sqrt{\tau}}\right) \\
& -\frac{1}{\sigma \sqrt{\tau}} e^{2 \mu y / \sigma^{2}} n\left(\frac{y+\mu \tau}{\sigma \sqrt{\tau}}\right) .
\end{aligned}
$$

We now expand the second term of (A3):

$$
\begin{aligned}
& \int_{-\infty}^{\ln \left(P_{0} / P_{t}\right)}\left(P_{0}-P_{t} e^{y}\right)\left\{\frac{1}{\sigma \sqrt{\tau}} n\left(\frac{-y+\mu \tau}{\sigma \sqrt{\tau}}\right)+\frac{2 \mu e^{2 \mu y / \sigma^{2}}}{\sigma^{2}}\right. \\
& \left.\cdot N\left(\frac{y+\mu \tau}{\sigma \sqrt{\tau}}\right)-\frac{e^{2 \mu y / \sigma^{2}}}{\sigma \sqrt{\tau}} n\left(\frac{y+\mu \tau}{\sigma \sqrt{\tau}}\right)\right\} d y \\
& =P_{0} N\left(\frac{\ln \left(P_{0} / P_{t}\right)-\mu \tau}{\sigma \sqrt{\tau}}\right) \\
& +P_{0}\left\{\left(\frac{P_{0}}{P_{t}}\right)^{2 \mu / \sigma^{2}} N\left(\frac{\ln \left(P_{0} / P_{t}\right)+\mu \tau}{\sigma \sqrt{\tau}}\right)\right. \\
& \left.-N\left(\frac{\ln \left(P_{0} / P_{t}\right)-\mu \tau}{\sigma \sqrt{\tau}}\right)\right\}+P_{0} N\left(\frac{\ln \left(P_{0} / P_{t}\right)-\mu \tau}{\sigma \sqrt{\tau}}\right) \\
& -P_{t} e^{\left(\mu+\sigma^{2} / 2\right) \tau} N\left(\frac{\ln \left(P_{0} / P_{t}\right)-\left(\mu+\sigma^{2}\right) \tau}{\sigma \sqrt{\tau}}\right)-P_{t}\left(\frac{\mu}{2 \sigma^{2}+\mu}\right) \\
& \cdot\left\{\left(\frac{P_{0}}{P_{t}}\right)^{\left(\sigma^{2}+2 \mu\right) / \sigma^{2}} N\left(\frac{\ln \left(P_{0} / P_{t}\right)+\mu \tau}{\sigma \sqrt{\tau}}\right)\right. \\
& \left.+e^{\left(\left(\sigma^{2} / 2\right)+\mu\right) \tau} N\left(\frac{\ln \left(P_{0} / P_{t}\right)+\left(\mu+\sigma^{2}\right) \tau}{\sigma \sqrt{\tau}}\right)\right\} \\
& -P_{t} e^{\mu+\left(\sigma^{2} / 2\right) \tau} N\left(\frac{\ln \left(P_{0} / P_{t}\right)-\left(\mu+\sigma^{2}\right) \tau}{\sigma \sqrt{\tau}}\right) .
\end{aligned}
$$

So, for $m_{T_{0}}^{t} \geqslant P_{0}$, letting

$d=\frac{\ln \left(P_{t} / P_{0}\right)+\left(\sigma^{2}+\mu\right) \tau}{\sigma \sqrt{\tau}}$

we have that

$$
\begin{gathered}
p^{P}\left(P_{t}, m_{T_{0}}^{t}, \tau\right) \\
=-P_{t} e^{\left(\left(\sigma^{2} / 2\right)+\mu-\rho\right) \tau} N(-d)+e^{-\rho \tau} P_{0} N(-d+\sigma \sqrt{\tau}) \\
+\frac{\sigma^{2} e^{-\rho \tau}}{\sigma^{2}+2 \mu} P_{t}\left\{\left(\frac{P_{0}}{P_{t}}\right)^{\left(\sigma^{2}+2 \mu\right) / \sigma^{2}} N\left(-d+\frac{\left(\sigma^{2}+2 \mu\right) \sqrt{\tau}}{\sigma}\right)\right. \\
\left.-e^{\left(\left(\sigma^{2} / 2\right)+\mu\right) \tau} N(-d)\right\},
\end{gathered}
$$


and a similar treatment of the first part of (A3) combined with the result from (A2) for $m_{T_{0}}^{t}<P_{0}$, letting

$d^{\star}=\frac{\ln \left(P_{t} / m_{T_{0}}^{t}\right)+\left(\sigma^{2}+\mu\right) \tau}{\sigma \sqrt{\tau}}$,

we have that

$$
\begin{aligned}
& p^{P}\left(P_{t}, m_{T_{0}}^{t}, \tau\right) \\
& =e^{-\rho \tau}\left(P_{0}-m_{T_{0}}^{t}\right)+m_{T_{0}}^{t} e^{-\rho \tau} N\left(-d^{\star}+\sigma \sqrt{\tau}\right) \\
& -P_{t} e^{\left(\left(\sigma^{2} / 2\right)+\mu-r\right) \tau} N\left(-d^{\star}\right)+\frac{\sigma^{2} e^{-\rho \tau}}{\sigma^{2}+2 \mu} \\
& \cdot P_{t}\left\{\left(\frac{m_{T_{0}}^{t}}{P_{t}}\right)^{\left(\sigma^{2}+2 \mu\right) / \sigma^{2}} N\left(-d^{\star}+\frac{\left(\sigma^{2}+2 \mu\right) \sqrt{\tau}}{\sigma}\right)\right. \\
& \left.-e^{\left(\left(\sigma^{2} / 2\right)+\mu\right) \tau} N\left(-d^{\star}\right)\right\} .
\end{aligned}
$$

\section{Acknowledgments}

The authors thank Montgomery Blair, director of science, Dollar Thrifty Automotive Group Inc., for motivating this research and providing access to data. Financial support for this research was received from the Natural Sciences and Engineering Research Council of Canada (NSERC) and the Mathematics of Information Technology and Complex Systems (MITACS).

\section{References}

Amram, M., N. Kulatilaka. 1999. Real Options: Managing Strategic Investment in an Uncertain World. Harvard Business School Press, Boston, MA

Anderson, C., H. Rasmussen, M. Davison. 2004. Revenue management: A real options approach. Naval Res. Logisit. 51(5) 686-703.

Anthes, G. H. 1998. The price had better be right. Computerworld 21 65-66.
Binkley, C. 2003. Marriott unveils best-rate pledge, offering discount. Wall Street J. (November 11).

Blair, M. 2002. Dynamic pricing: The new diversity of revenue management. IFORS Triennial Meeting, Edinburgh, Scotland.

Blair, M., C. Anderson. 2002. Performance monitor. J. Revenue Pricing Management 1(1) 57-66.

Carroll, W., R. Grimes. 1995. Evolutionary changes in product management: Experiences in the car rental industry. Interfaces 25(5) 84-104.

Conze, A., R. Viswanathan. 1991. Path dependent options: The case of lookback options. J. Finance 46(5) 1893-1907.

Cooper, T. 1986. Most-favored-customer pricing and tacit collusion. RAND J. Econom. 17(3) 377-388.

Cross, R. 1995. An introduction to revenue management. Handbook of Airline Economics. D. Jenkins, C. Ray, eds. McGraw-Hill, New York, $443-458$

Datamonitor. 2003. United States car rental. Industry report, Datamonitor Industry Profiles.

Dixit, A. K., R. S. Pindyck. 1994. Investment Under Uncertainty. Princeton University Press, Princeton, NJ.

Geraghty, M., E. Johnson. 1997. Revenue management saves National Car Rental. Interfaces 27(1) 107-127.

Goldman, M., H. Sosin, M. Gatto. 1979. Path dependent options: Buy at the low, sell at the high. J. Finance 34(5) 1111-1127.

Harrison, J. M. 1985. Brownian Motion and Stochastic Flow Systems. John Wiley and Sons, New York.

Hull, J. C. 2003. Options, Futures and Other Derivatives, 5th ed. PrenticeHall, Upper Saddle River, NJ.

Kimes, S. 1989. Yield management: A tool for capacity constrained service firms. J. Oper. Management 8 348-363.

Merton, R. C. 1990. Continuous-Time Finance. Blackwell, Cambridge, MA.

Miller, W. 1999. Airlines take to the Internet. Industry Week 248(15) 130-134.

Png, I. 1991. Most-favored-customer protection versus price discrimination over time. J. Political Econom. 99(5) 1010-1028.

Smith, B. C., J. F. Leimkuhler, R. M. Darrow. 1992. Yield management at American Airlines. Interfaces 22(1) 8-31.

Trigeorgis, L. 1996. Real Options: Managerial Flexibility and Strategy in Resource Allocation. MIT Press, Cambridge, MA.

Wilmott, P. 2000. Paul Wilmott on Quantitative Finance. John Wiley and Sons, Chichester, UK. 